EPJ Web of Conferences 59, 16001 (2013)

DOI: $10.1051 /$ epjconf/20135916001

(C) Owned by the authors, published by EDP Sciences, 2013

\title{
High energy density physics studies using intense particle beams at the FAIR facility at Darmstadt
}

\author{
N.A. Tahir ${ }^{1, a}$, A. Shutov ${ }^{2}$, A.R. Piriz ${ }^{3}$, C. Deutsch ${ }^{4}$ and Th. Stöhlker ${ }^{1}$ \\ 1 GSI Helmholtzzentrum für Schwerionenforschung Darmstadt, Planckstrasse 1, \\ 64291 Darmstadt, Germany \\ 2 IPCP Cherogolovka, Russia \\ 3 E.T.S.I. Industriales, Universidad de Castilla-La Mancha and Instituto de Investigaciones \\ Energéticas, 13071 Ciudad Real, Spain \\ 4 LPGP, Université Paris-Sud, 91405 Orsay, France
}

\begin{abstract}
High Energy Density (HED) physics spans over numerous areas of basic and applied physics, for example, astrophysics, planetary physics, geophysics, inertial fusion and many others. During the past fifteen years, great progress has been made on the development of bunched intense particle beams that have emerged as a novel tool for studying HED physics. In this paper we present two experiment designs that have been worked out for HED physics studies at the Facility for Antiprotons and Ion Research (FAIR) at Darmstadt. This facility has entered into construction phase and will provide one of the largest and most powerful particle accelerators in the world.
\end{abstract}

\section{INTRODUCTION}

Over the past decade, extensive theoretical work has been carried out to study the problem of generating High Energy Density (HED) matter in the laboratory employing intense heavy ion beams [1-5]. Several experiment designs that propose generation of HED matter considering isochoric and uniform heating of solid targets as well as shock compression of matter, have been proposed. The beam parameters used in this study correspond to those which are expected to become available at the new powerful synchrotron SIS100, that is being constructed within the framework of the Facility for Antirptons and Ion Research (FAIR) at Darmstadt. When the SIS100 becomes fully operational, it will deliver a uranium beam with an intensity (total number of particles per bunch), $\mathrm{N}=5 \cdot 10^{11}$ ions and a wide range of particle energy $(400 \mathrm{MeV} / \mathrm{u}-2.7 \mathrm{GeV} / \mathrm{u})$ will be available. The bunch length, $\tau$ for the above energy range will be 100-25 ns while the repetition rate of the accelerator will be $1 \mathrm{~Hz}$. The focal spot geometry will be circular, nevertheless, provisions are being made for an annular shaped focal spot to carry out compression experiments. An rf-wobbler is being designed to generate the latter focal spot geometry [5, 6]. Earlier, another option, namely, use of a plasma lens, was considered for this purpose [7], however, this option has now been dropped.

It is to be noted that particle beams provide great flexibility to the experimentalists as they are capable of generating HED matter by two very different dynamic techniques, namely, by shock compression and isochoric heating of matter. In this paper we present design of two different experiments that are based on the shock compression technique. The results are presented in the next section.

\footnotetext{
${ }^{a}$ e-mail: n.tahir@gsi.de
}

This is an Open Access article distributed under the terms of the Creative Commons Attribution License 2.0, which permits unrestricted use, distribution, and reproduction in any medium, provided the original work is properly cited. 

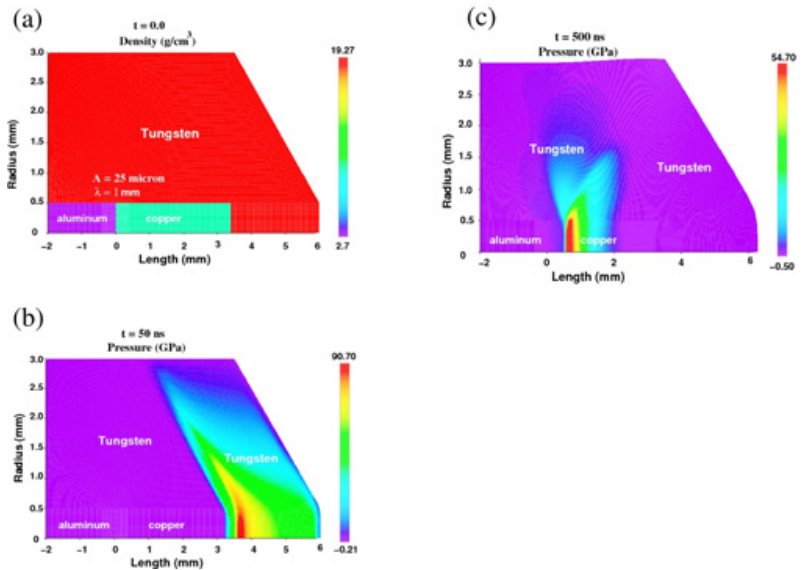

Figure 1. (a) Beam-target geometry for generating a plane steady shock wave using a Mach type reflection scheme; (b) pressure distribution at $\mathrm{t}=50 \mathrm{~ns}, \mathrm{~N}=5 \times 10^{10}$ ions / bunch; (c) pressure distribution at $\mathrm{t}=500 \mathrm{~ns}$.

\section{NUMERICAL SIMULATION RESULTS}

In this section we present the numerical simulation results that have been obtained using a twodimensional hydrodynamic code, BIG2 [8].

\subsection{Generation of plane shocks using a Mach type reflection scheme}

We have proposed a design that uses a Mach type reflection scheme to produce a plane stationary shock front using ion beams [4].

The beam-target set up is shown in Fig. 1(a) which is a multi-layered conical shaped cylindrical target that is irradiated by the beam from the right side. First we consider a lower beam intensity of $5 \times 10^{10}$ ions per bunch. The pressure produced in the target at $\mathrm{t}=50 \mathrm{~ns}$ (end of the ion bunch) is presented in Fig. 1(b). It is seen that the parallel ion beam deposits maximum energy density in the conical region at the location of the Bragg peak. This leads to an inward and an outward moving shock wave. The inward moving shock wave, after reflection at the axis of symmetry, forms a Mach configuration [9]. This leads to an extended, steady and plane shock front with a pressure of about $55 \mathrm{GPa}$, as shown in Fig. 1(c) where we present the pressure distribution at $\mathrm{t}=500 \mathrm{~ns}$.

To simplify the calculations, the inner part of the target where the shock front exists, is magnified and is presented in Fig. 2(a). It is seen that at $\mathrm{t}=500 \mathrm{~ns}$, the shock front is still in the $\mathrm{Cu}$ region, but is close to the $\mathrm{Cu}-\mathrm{Al}$ interface that is located at $\mathrm{L}=0$. The pressure in the shock front is about $55 \mathrm{GPa}$ while the temperature in the shock heated $\mathrm{Cu}$ is around $900 \mathrm{~K}$ which means that the material is still in solid state and possesses solid constitutive properties.

If one uses the highest beam intensity of $5 \times 10^{11}$ ions / bunch, the shock is driven by a much higher pressure of about $480 \mathrm{GPa}$ and the temperature in the shocked $\mathrm{Cu}$ is of the order of $18000 \mathrm{~K}$ that corresponds to a fluid state. Moreover, in this case the shock moves faster and arrives close to the $\mathrm{Cu}-\mathrm{Al}$ interface at an earlier time of about $250 \mathrm{~ns}$.

This scheme has been applied to study the growth of the Richtmyer-Meshkov instability in the linear and the non-linear regimes for solids as well as for ideal fluids by introducing an initial perturbation at the $\mathrm{Cu}-\mathrm{Al}$ boundary as presented below. Further details can be found in [4]. 


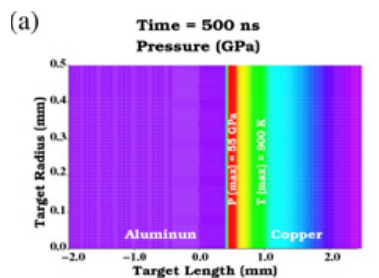

(b)

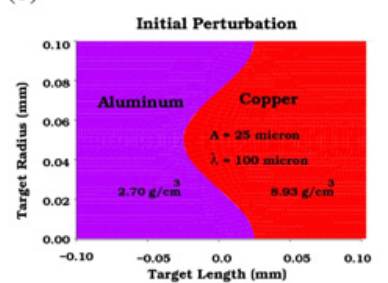

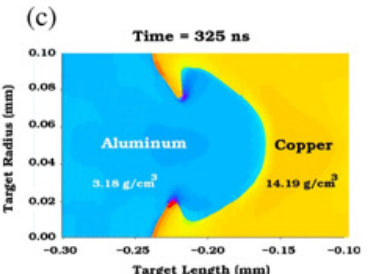

(d)

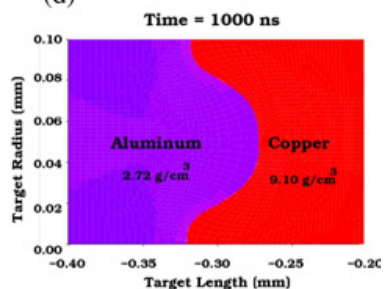

Figure 2. (a) Magnified view of the shock region close to the interface; (b) $\lambda=100 \mu \mathrm{m}$ and initial $\mathrm{A}=25 \mu \mathrm{m}$; (c) amplitude at $\mathrm{t}=325 \mathrm{~ns}$ in an ideal fluid $\left(\mathrm{N}=5 \times 10^{11}\right.$ ions/bunch); (d) amplitude at $\mathrm{t}=1000 \mathrm{~ns}$ in solid $\left(\mathrm{N}=5 \times 10^{10}\right.$ ions/bunch).

\subsection{Richtmyer-Meshkov growth studies}

To study the $\mathrm{RM}$ instability growth across the $\mathrm{Cu}-\mathrm{Al}$ interface in an ideal fluid (using a beam intensity of $5 \times 10^{11}$ ions / bunch), a perturbation with wavelength, $\lambda=100 \mu \mathrm{m}$ having an initial amplitude, $\mathrm{A}=25 \mu \mathrm{m}$ is applied in the vertical direction. The corresponding wave number, $\mathrm{k}=6.28 \times 10^{2} \mathrm{~cm}^{-1}$ so that the product $\mathrm{kA}=1.57$, which represents a non-linear regime. Since the interface has a radius of $0.5 \mathrm{~mm}$, five wavelengths will fit in that region. However, for the simplicity of the calculations, we study the behavior of a single wavelength as shown in Fig. 2(b). The shock travels from right to left (from copper into the aluminum).

As shock propagates from a high-density medium $(\mathrm{Cu})$ to a low-density medium (Al), the perturbation phase is inverted. This is clearly seen in Fig. 2(c) which shows a typical non-linear "mushroom type" growth. The perturbation amplitude continues to grow as no stabilizing mechanism exists.

In Fig. 2(d), we plot results of instability growth in solid target which shows that at $\mathrm{t}=1000 \mathrm{~ns}$, the perturbation phase has been inverted, but the amplitude is comparable to the initial amplitude of $25 \mu \mathrm{m}$ and there is no mushroom shaped growth and the perturbation stabilizes due to the constitutive properties of the material.

Simulations using smaller perturbation amplitude of $1 \mu \mathrm{m}$ corresponding to a linear regime, have shown that initially the amplitude grows linearly in case of an ideal fluid, but at a later stage it enters into the non-linear regime and loses its sinusoidal structure. In case of a solid, on the other hand, small perturbations do not grow at all but are completely stabilized due to the solid constitutive properties.

\subsection{Multiple reflection of cylindrical shocks and the LAPLAS scheme}

Numerical simulations have shown that an intense ion beam can be employed to generate a low-entropy compression that leads to physical conditions that are expected to exist in the interiors of the Giant planets in our solar system as well as in the exoplanets. This type of experiment is named LAPLAS [LAboratory PLAnetary Science]. In fact there are two different LAPLAS schemes shown in Figs. 3(a) and 3(b) respectively. In both cases, the sample material (frozen hydrogen or water) is enclosed in a thick shell of a high-Z material and the target is irradiated face-on. The ion energy is chosen in such a way that the ion range is larger than the target length so that the Bragg peak does not lie inside the target and the energy deposition is uniform along the axial direction. It is seen that in case of Fig. 3(a), one 
(a)

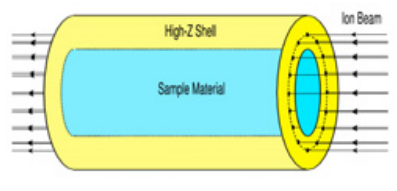

(b)

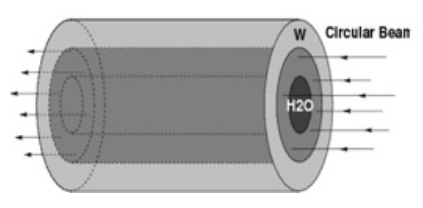

(c)

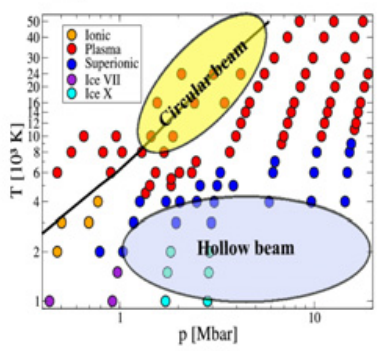

Figure 3. (a) Beam-target configuration using an annular focal spot; (b) beam-target configuration using a circular focal spot; (c) each colored point corresponds to a QMD simulation in thermodynamic equilibrium. When the electronic conductivity exceeds $100 /(\mathrm{Ohmcm})$ [10], the ionic (dissociated) fluid is labeled as plasma. The solid line is the principal Hugoniot curve.

uses a beam with an annular focal spot so that the sample material is not directly heated by the projectile ions and the energy is deposited only in the surrounding high $-\mathrm{Z}$ shell. This leads to an envelop of high pressure around the sample that drives a shock inwards along the radius. This shock reverberates between the cylinder axis and a low entropy compression of the sample material is achieved. Simulations have shown that using the SIS100 parameters, it is possible to compress solid hydrogen to a density of about $3 \mathrm{~g} / \mathrm{cm}^{3}$ having a pressure of the order of 30 Mbar while the temperature remains low (about $10000 \mathrm{~K}$ ) which are conditions expected at the interiors of the Gaint palnets. This experiment can also help to understand hydrogen metallization [10] problem.

In case of the beam-target scheme shown in Fig. 3(b) that uses a circular beam, the sample material is also directly heated by the ion beam and one achieves a hydrogen density of $1.2 \mathrm{~g} / \mathrm{cm}^{3}$, a pressure of about $11 \mathrm{Mbar}$ and a temperature of the order of $5 \mathrm{eV}$. These physical conditions also represent the physical state in the interiors of hydrogen rich Giant planets.

Simulation results of compression of water are summarized in Fig. 3(c) while details can be found in [3]. It is seen that with a circular focal spot, one achieves a plasma state only. Using an annular focal spot, on the other hand, it is possible to access the theoretically predicted super ionic state of water [11]. Similar work on planetary physics studies is reporte elsewhere $[12,13]$.

\section{References}

[1] N.A. Tahir et al., Phys. Rev. E 63, 016402 (2001)

[2] N.A. Tahir et al., Phys. Rev. Spec. Topics Beams Accel. 6, 020101 (2003)

[3] N.A. Tahir et al., N. J. Phys. 12, 073022 (2010)

[4] N.A. Tahir et al., Phys. Plasmas 18, 032704 (2011)

[5] A.R. Piriz et al., Plasma Phys. Controlled Fusion 45, 1733 (2003)

[6] S. Minaev et al., Nucl. Instr. Meth. A 620, 99 (2010)

[7] U. Neuner et al., Laser Part. Beams 85, 4518 (2000)

[8] V.E. Fortov et al., Nucl. Sci. Eng. 123, 169 (1996)

[9] G. Ben-Dor, Shock Wave Reflection Phenomena, Springer (2007)

[10] W.J. Nellis, Rep. Prog. Phys. 69, 1479 (2006)

[11] T.R. Mattsson and M. Desjarlais, Phys. Rev. Lett. 97, 017801 (2006)

[12] C. Cavazzoni et al., Science 283, 44 (1999)

[13] P. M. Celliers et al., Phys. Plasmas, 11 L41 (2004) 